\title{
Fast Radiation Dynamics during ELMs in TCV
}

\author{
G. Veres ${ }^{1}$, R.A. Pitts ${ }^{2}$, A. Aranyi ${ }^{1}$, A. Bencze ${ }^{1}$, M. Berta ${ }^{1}$, B. Gulejova ${ }^{2}$, S. Kálvin ${ }^{1}$ \\ and the TCV Team ${ }^{2}$ \\ ${ }^{1}$ KFKI - RMKI, Association EURATOM-HAS, H-1525, Budapest, Hungary \\ ${ }^{2}$ CRPP-EPFL, Association EURATOM-Confédération Suisse, CH-1015 Lausanne, Switzerland
}

Introduction. Despite significant progress in their understanding, ELM (Edge Localised Mode) instabilities remain a serious concern for future fusion devices (e.g. ITER). This is primarily due to the local transient heat loads that such events provoke on plasma-facing surfaces. Whilst the heat loads themselves can be measured sensitively on fast timescales using IR thermography, the plasma radiation distribution during the ELM event and in response to the plasma-wall interaction is harder to study with high time resolution. Such data can make an important contribution to understanding of the ELM physics. This paper reports on new measurements in TCV of radiation during the ELM cycle on the microsecond timescale.

With the advent of compact, multichannel photodiode arrays - sensitive from visible/near UV to soft X-ray wavelengths - it has become possible to study the redistribution of radiation during fast transient events, such as ELMs and disruptions. Compared to traditional foil bolometers, these photodiodes offer excellent time resolution (their rise time is well below $1 \mu \mathrm{s}$ ) but at the price that the spectral sensitivity of the diode is wavelength dependent. Multiple camera systems consisting of several photodiode arrays and allowing for radiation detection along several lines of sight, enable tomographic inversion of the measured signal and the computation of the $2 \mathrm{D}$ radiation distribution. Just such an array of pinhole cameras has recently been installed on TCV [4] providing high spatial resolution across the whole poloidal cross-section.

Any tomographic algorithm introduces distortions to the images obtained using it. The effect of these distortions and the influence of the random measurement noise and uncertainty that accompanies any measurement can be quantified with Bayesian statistical methods. This basically means that the probable emissivity distribution, together with the range of uncertainty, can be determined from the tomographic measurement. Put another way, this means that the derived 2D radiation distributions are objective and quantitative.

Experimental. Our measurements have been made in TCV single null lower discharges heated with $2 \mathrm{MW}$ of third harmonic ECRH $\left(\mathrm{I}_{\mathrm{p}}=360 \mathrm{kA}, \overline{\mathrm{n}}_{\mathrm{e}}=4 \cdot 19 \mathrm{~m}^{-3}, \mathrm{~B}_{\mathrm{t}}=1.45 \mathrm{~T}\right.$ with the ion gradB drift directed towards the X-point). These ELMy H-mode plasmas are characterized by an ELM frequency of about $40 \mathrm{~Hz}$, with each ELM expelling plasma stored energy in the range of several $\mathrm{kJ}(\approx 10 \%$ of the total stored energy). Although a strict ELM classification has not yet been possible, the ELMs are very probably of Type I [5].

A new array of fast radiation detectors based on AXUV diodes (manufacturer: International 
Radiation Detectors [2]) has recently come into operation on the TCV tokamak [3, 4]. It comprises 7 individual cameras providing 140 lines of sight encompassing the entire poloidal cross-section and is equipped with amplification and acquisition electronics permitting time resolution up to 5 $\mu$ s. The system can be used to perform tomographic inversions of total radiation emission with unprecedented speed - orders of magnitude faster than traditional foil bolometers and with good spatial resolution.

Tomographic reconstructions of AXUV diode signals have been obtained using serial expansion constrained optimization with regularization using local base functions [6, 7]. Based on these reconstructed profiles, the time dependence both of the total radiative power emitted by the plasma during an ELM and the radiation emitted in different plasma regions have been determined.

The $2 \mathrm{D}$ radiation image distributions presented in this paper were obtained by inverting the signals recorded during a single ELM. Due to the excellent signal-to-noise ratio there is no need to conditionally average the signals over many ELMs.

In the analysis the diode wavelength dependent sensitivity causes interpretation difficulties. Overcoming this problem is very difficult, if not impossible without additional spectral measurements of the target plasma, so here all measurement channels are assumed to have the same spectral sensitivity. In this case the result of the tomographic reconstruction is the radiation in the spectral range defined by the detector sensitivity.

Results and Discussion. Tomographic reconstructions on the several microsec timescale reveal an ELM radiation cycle characterised by distinct phases (see Figure 1.). The result of the Bayesian analysis shows that the pre-ELM radiation has a definite asymmetry - the radiation is $30 \%$ higher around the X-point then elsewhere, as would be expected from the cooler plasma in the X-point vicinity (providing optimum conditions for impurities to radiate), together with expanded flux surfaces (providing long residence times for impurities in this region). At the ELM onset the radiating zone around the X-point begins to move towards divertor strike points (or alternatively the radiation simply begins to increase around the strike points) and simultaneously a strong radiating belt appears in the vicinity of the outboard midplane wall with lobes distributed above and below the midplane. This is a direct indication of the arrival of ELM filaments at the wall, where the separatrix wall gap is only $6 \mathrm{~cm}$ for this discharge. The outboard midplane radiation disappears $\sim 50$ $\mu$ s later but the enhanced divertor radiation persists for several $100 \mu$ s before the radiation front moves away from the targets to recover its pre-ELM distribution. Inspection alone of the outboard midplane radiation belt is insufficient to determine the precise origin of the light emission, which can be due to both the light of the plasma itself and/or the result of emission due to deuterium and impurity recycling provoked by the ELM impact.

In addition to the strong midplane interaction, an interesting further phenomena is the appearance of an event in the X-point region which precedes the arrival of filaments at the outboard wall. 
The continuous radiating zone inside the separatrix close to the $\mathrm{X}$-point seems to split into two distinct regions, with the one closest to the inner wall moving towards the inner strike point. Unfortunately, as before, without additional spectral information it is impossible to unambiguously determine the origin of this radiation increase: it can be due to both an increase in local density or a change in the photonic excitation function (ie a change in plasma temperature).

Figure 2a shows the time evolution of the total radiated power emitted by the plasma in different regions. The uncertainty of the determined radiation power is 3-5\% and the pre-ELM emissivities have been subtracted. The radiation begins to increase first around the X-point, then at the LFS midplane and HSF strike point, and finally at the outer strike point.

An explanation for the radiation activity in the X-point region prior to the arrival of the ELM filaments at the outboard wall is not clear. In [8] a so-called peeling-tearing instability is suggested as being responsible for some ELM activity. This instability is most unstable just in the $\mathrm{X}$-point region, because along the separatrix away from the $\mathrm{X}$-point the ideal and resistive MHD external kink/peeling modes, due to the diverging q, are stable. Since in the development of the peeling-tearing instability finite plasma resistivity is crucial, we can speculate that the increase of the radiative power indicates a drop in the plasma temperature (TCV is a carbon dominated machine and the cooling curve of carbon has a negative slope characteristic of the X-point region), which in turn would increase the resistivity. Further analysis will be undertaken to rule out specific contributors to this X-point radiation by using Mirnov coil, Langmuir probe and soft X-ray data.

\section{References}

[1] R.L. Boivin, J.A. Goetz, E.S. Marmar et al., Rev. Sci. Instrum. 70 (1999) 260.

[2] http://www.ird-inc.com

[3] A.W. Degeling, H. Weisen, A. Zabolotsky et al., Rev. Sci. Instrum. 75 (2004) 4139.

[4] G. Veres, R.A. Pitts, M. Wischmeier et al. J. Nucl. Mat. 363-365 (2007) p. 1104-1109.

[5] L. Porte et al., accepted for publication in Nucl. Fusion.

[6] S. Zoletnik, S. Kálvin, Rev. Sci. Instrum. 64 (1993) 1208.

[7] L.C. Ingesson, B. Alper, H. Chen et al., Nucl. Fusion 38 (1998) 1675.

[8] G.T.A. Huysmans, Plasma. Phys. Contr. Fusion 47 (2005) 2107. 

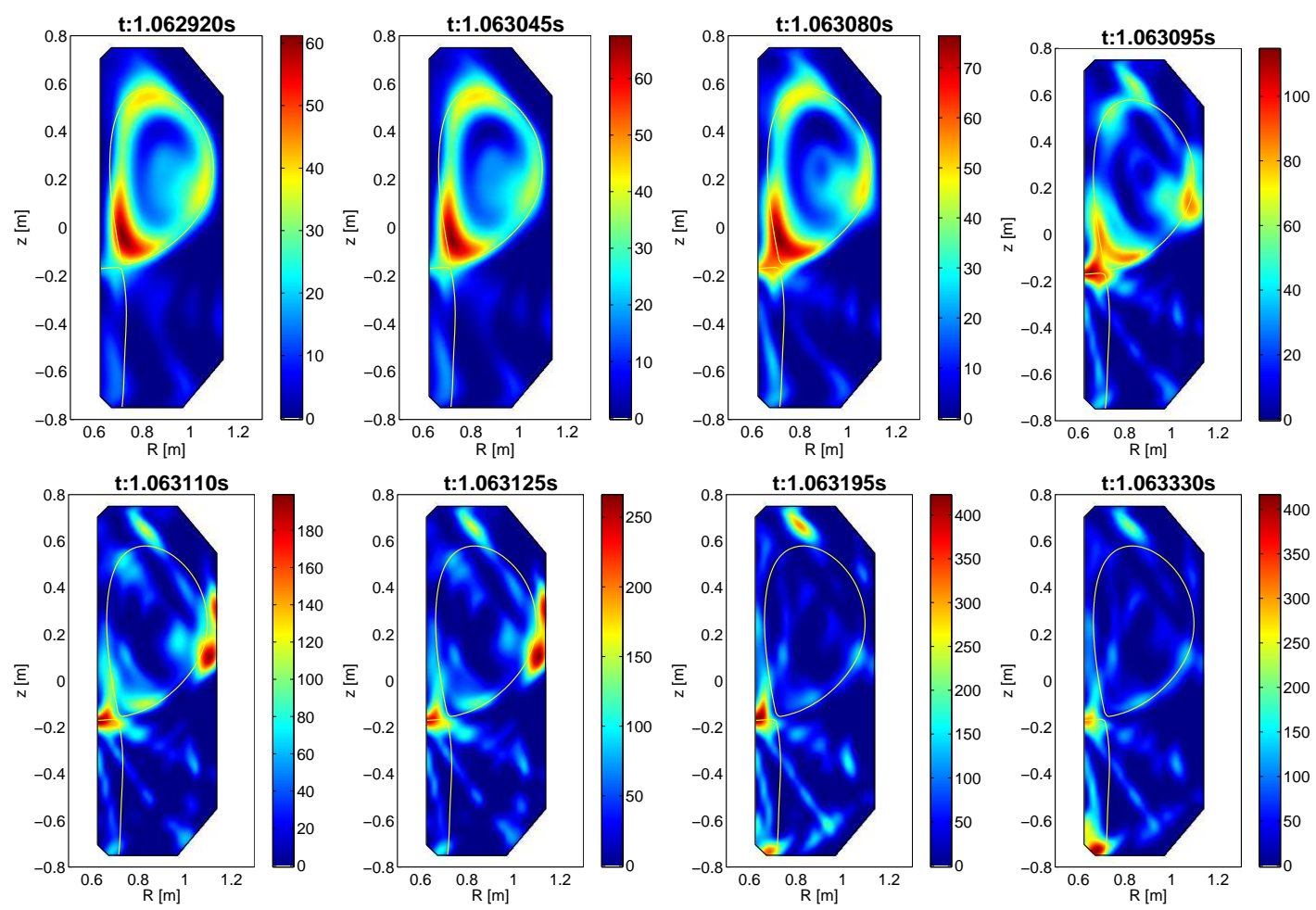

Figure 1: Total radiative emissivities during and ELM taken at times indicated on Fig. 2. Powers are given in $\mathrm{kW} / \mathrm{m}^{3}$.
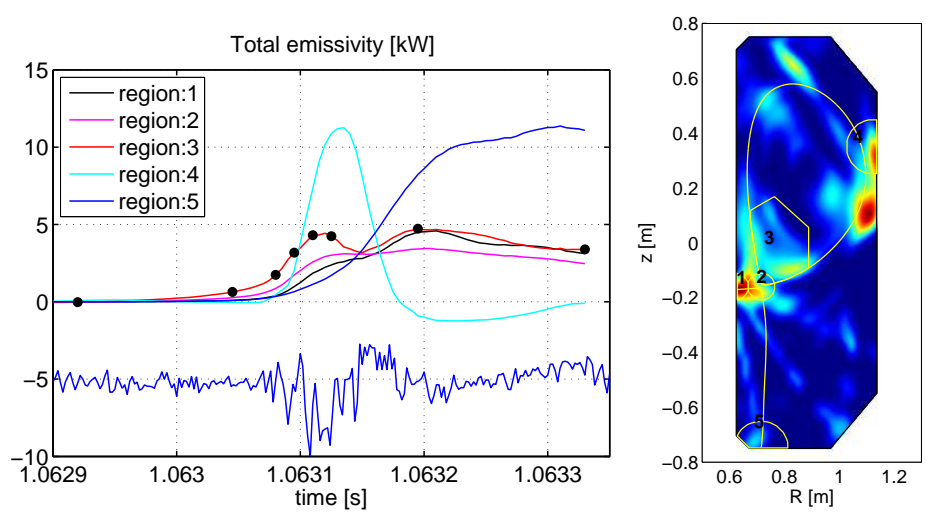

Figure 2: a) Total radiative powers emitted by different plasma regions during an ELM cycle. The points indicate the times, when the images of Fig.l are taken. In the lower part of the Figure the Mirnov activity in the same toroidal section is shown. $b$ ) The explanation of the regions of plot a). 